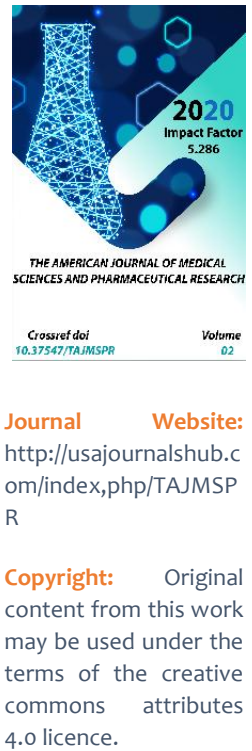

\title{
Epidemiology, Risk Factors, Clinical Current, Diagnostics, Principles Of Treatment And Prevention Of Acute Myocardial Infarction In The Elderly And Old Population (Review)
}

\author{
Rakhmatova Dilbar Baxriddinovna \\ Bukhara State Medical Institute, Bukhara, Uzbekistan
}

\section{ABSTRACT}

In view of the special medico-social importance of cardiovascular diseases, their prevention is one of the most important tasks facing medical science and the health care system. Ischemic heart disease and its most serious complication, acute myocardial infarction, is the main and most dangerous disease of the cardiovascular system. Epidemiological studies and screening measures for the prevention of cardiovascular diseases contain scientific data that can not only reduce the incidence, but, undoubtedly, prolong the life of the elderly and senile population.

\section{KEYWORDS}

Cardiovascular diseases, ischemic heart disease, acute myocardial infarction, epidemiology, prevention.

\section{INTRODUCTION}

Cardiovascular diseases are the leading cause of death in our country, accounting for more than $56 \%$ of all deaths. In addition, cardiovascular diseases have not only in terms of medical, but also social and economical importance because they are associated with early death, i.e., the death of people of working age. [26; 27] Traditionally, system of prevention of the cardiovascular diseases, especially the acute myocardial infarction should be based on the identification of variable risk factors in the elderly groups of the population. Such acute forms and conditions of ischemic heart disease, the 
presence of each of them increases the likelihood of death caused by cardiovascular diseases. [22; 28] In recent decades, cardiovascular prophylaxis has been actively developed in a small group of elderly patients, as well as prevention algorithms and rehabilitation programs were created. Almost $3 / 4$ death occurs from ischemic heart disease among people elder than 65 , almost $80 \%$ of deaths from acute myocardial infarction occur in elderly patients belong to this age group. [20;29] In older age groups prevention and treatment of acute myocardial infarction are specific and are associated with the rapid occurrence of co-morbidities, which leads to restrictions on the use of primary, secondary and tertiary prevention methods. This problem can be solved with the help of special epidemiological programs, which can help to emanate the epidemiological situation related to the prophylaxis of acute myocardial infarction in a particular region in a precise way. [1;33;34] In order to detect acute myocardial infarction early and completely and to prevent "targetedly" there should be conducted mass epidemiological surveys of the elderly people. In Uzbekistan, as in most countries, acute myocardial infarction develops among men after the age of 45 and women after the age of 55. [30]

\section{MATERIALS AND METHODS}

Analysis of epidemiology, the incidence of risk factors, the principles of clinical course, diagnosis, treatment and prevention of acute myocardial infarction in the elderly people.

\section{RESULTS AND DISCUSSION}

The incidence of cardiovascular diseases in Kazakhstan shows their growth, this is also observed in European and Central Asian countries. Currently, the impact of cardiovascular diseases on the level of health of the entire world population is forcing the entire scientific community to search and find new ways to solve this problem. [1] According to the World Health Organization's Register of Acute Myocardial Infarction long-term (19842016) dynamics of the main epidemiological indicators of acute myocardial infarction (AMI) 20 Standard diagnostic research (clinical, electrocardiographic, biochemical, 49,606 cases of suspected acute myocardial infarction were recorded during the analysis using pathomorphological criteria of which 30,362 (61.2\%) patients confirmed the disease. The overall mortality rate in patients with acute myocardial infarction in Tomsk 35.98\% and has not undergone any changes, but since 2000 , the total number of deaths due to hospitalization there is an upward trend in mortality. [2] Recurrent, i.e. recurrent myocardial infarction development is important risk factors include those over 60 years of age, comorbid pathologies, and treatment modalities. development risk assessment and features of the formation of important databases / registers, issues related to the selection of the analyzed indicators were discussed. [3] During the thirteen-year follow-up period to assess the dynamics of obesity phenotypes and their relationship to the risk of myocardial infarction (MI) The study was conducted on the basis of the international project HAPIEE (number of men and women aged 45-69, $\mathrm{n}=9360,2003-2005$ ). Body weight index (BWI)> $30 \mathrm{~kg} / \mathrm{m2}: 3197$ people; 857 men (26.8\%) and 2340 women 
(73.2\%) were analyzed. The analysis was performed in a population with a metabolically healthy obesity phenotype (MHOP). The MHOP situation has been volatile for 13 years more than half of men and women have switched to the metabolic unhealthy obesity phenotype (MUOP). MUOP the relative risk of myocardial infarction was significantly higher in the MUOP group than in the MHOP group. [4] The life of patients treated with modern methods of myocardial infarction observed in patients with type 2 diabetes increases the duration. However, it is more common in patients with type 2 diabetes than in those without diabetes myocardial infarction is 3 times more complicated with acute left ventricular failure (ALVF) and lethality in non-type 2 diabetics was twice as high. [5] Old age is the most important and independent form of fibrillation ( $F$ ) is a risk factor. The proportion of $\mathrm{BF}$ in patients aged $65-85$ years reaches $70 \%$, and the average age of patients with BF is 75 years. forms. Therefore, the risk of thromboembolic and hemorrhagic complications in the elderly, as well as antithrombotic the approach to therapy should be evaluated. [6] Supported by the Taiwan National Health Insurance Research Institute A study from the Longital Health 2005 (LHID 2005) database found that influenza (IV) in the elderly compatible or incompatible strains of vaccines are useful in reducing the risk of acute myocardial infarction (acute myocardial infarction) studied. All participants were 65 years of age or older. An elderly risk model to assess the severity of an acute myocardial infarction used. The study involved 93,051 affected and 109,007 untreated people. Selected in men exposed to appropriate vaccines (in 2008), the number of heartbeats changed significantly (heart rate (HR)-0.681; 95\% confidence interval 0.509-0.912), and women's HR almost unchanged (HR - 0.737; 95\% confidence interval 0.527-1,029). In 65-yearolds, the risk of acute myocardial infarction is particularly high in men if the strains of influenza (IV) vaccines are compatible as long as it can be reduced. [7] The role of heredity in the development of ischemic heart disease and myocardial infarction is known, however the mechanisms for its implementation have not yet been fully explored. In the pathogenesis of atherosclerosis, in the regulation of hemostasis, there are many genes responsible for the synthesis of proteins involved in inflammatory reactions did not bring significant success in research. The main problem of this study is the results obtained the level of reproduction was very low. A thorough study of the human genome opens up new perspectives in the study of this problem explains. [8] Blood clotting is a biochemical reaction that is activated when the integrity of the vascular system is compromised is a complex network in which the purpose of blood clotting is to convert blood plasma from a liquid state to a jelly-like state, and as a result to stop the bleeding. Any disturbances in the delicate balance of this system are associated with thrombosis or bleeding leads to serious consequences. [9] Risk factors for cardiovascular complications in patients over 60 years of age Influence of the age factor on the characteristics of the "Hospital for War Veterans" in Tyumen in 20132014 studied in 146 patients older, clinical, laboratory methods, target levels as research methods a survey was conducted to identify risk factors for cardiovascular disease. Patients 180 and $110 \mathrm{mmHg}$ of blood pressure in $98 \%$. found to be higher than A leading factor in the development of atherosclerosis dyslipidemia occurs in $63.7 \%$ of patients over 
60 years of age. According to INTERHEART checks, active Smoking risk factors are rare $6 \%$. Hypodynamics is one of the leading causes of premature death has been detected in $60 \%$ or more of patients over 60 years of age. Fruits and including a shortage of vegetables, which was estimated at $12.9 \%$. The next leading cause of premature death is alcohol consumption - $11.9 \%$. Patients over the age of 60 do not consume alcohol in $88.7 \%$ of cases. Interestingly, it's long $100 \%$ of survivors do not consume alcohol, and in elderly and elderly patients this figure is $52.4 \%$ and $50 \%$, respectively. was determined accordingly. [10] ST-segment after myocardial infarction and primary percutaneous-coronary intervention in order to study the main clinical features of patients with different conditions of obesity after ascension 105 men with myocardial infarction and primary percutaneous coronary intervention with ST-segment elevation selected. Statistical analysis the first clinical study in a group of patients studied using the Statistics 10 software package indicators were found to be related to general obesity and epicardial obesity and their various combinations. Coronary atherosclerosis, myocardial infarction 2-3 times higher in groups with ES compared with groups without ES. Identified life-threatening arrhythmias in acute myocardial infarction, leptin resistance, albuminuria, etc. Thus, there is no paradox of obesity, but the criteria for assessing the degree and type of obesity there is a paradox. [11;33;34] Cardialgias in a number of diseases not related to heart disease when discussed, the main emphasis was on the clinical course of psychogenic cardialgias. He had psychosomatic pathology the use of voluntary psychotherapy in the complex treatment of patients certainly has a positive clinical effect, which leads to rapid cardialgia stops or reduces the frequency and intensity of pain syndrome. [12] Atypical acute myocardial infarction among patients observed in $23.5 \%$ of cases, mainly in older people on the background of arterial hypertension, obesity and diabetes observed. In rare cases of atypical acute myocardial infarction, changes in the pre-infarction state are called angina pectoris. Alternating with intensification and cessation, but the important aspect in this category of patients is that they are medical. Help is often sought with "nonspecific complaints" before or during the development of myocardial infarction. Nonspecific complaints: shortness of breath, weakness, high blood pressure in the last 4 weeks, cardiovascular disease Compulsory ECG in elderly and elderly patients with type 2 diabetes mellitus, as well as it is necessary to determine the activity of specific cardio-specific enzymes in the serum of patients, because atypical acute myocardial infarction Only a comprehensive diagnostic approach allows timely diagnosis of myocardial infarction. [13] Multifocal The role of IL-18 in the formation of atherosclerosis was determined, and on the 12th day of hospitalization IL-18 high concentration - an increase in total cholesterol and low-density lipoprotein (LDL), as well as left associated with a decrease in the force of contraction of the ventricular myocardium. Multifocal atherosclerosis (MFA) and ST-segment 1.5-fold increase in IL-18 levels in patients with concomitant myocardial infarction recorded. [14] According to the literature, $40-60 \%$ of patients with acute myocardial infarction are obstructive vascular coronary artery damage and nonobstructive multi-vessel coronary artery damage in $8.8 \%$ of patients. [15] The diagnosis of recurrent myocardial infarction is usually very difficult. Recurrent myocardial 
infarction is atypical, possibly fatigue, shortness of breath, epigastrium, pain in the hand. Recurrent myocardial infarction is more difficult than new infarction. In some cases, recurrent infarction does not appear on the ECG at all. (Chernov A.3., Kechker M.I., 1979). At this time, the comparison ECG, daily monitoring, echocardiography and, of course, immunological, biochemical analysis of blood creatine phosphokinase, myoglobin, troponin I and lactate dehydrogenase isomer, MVCPhK, LDG 1, AST, ALT and other specific cardiac markers an increase or decrease in blood may help. [16] This problem in Russia and around the world at high rates of acute myocardial infarction accompanied by elevation of the ST segment, which determines the urgency prevalence and mortality. According to the World Health Organization (WHO), 7.4 in 2018 Millions die of coronary heart disease (CHD) and myocardial infarction is the highest percentage of deaths. [17] There were 627 cases of acute myocardial infarction in Tomsk in 2008 and 840 in 2013 when comparing the duration of the prehospital period (DD) in patients with $A M I$, the study analysis showed that Indications for acute myocardial infarction are the duration of the $\mathrm{AMI}$ index and the timeliness of patients in need of medical care does not affect their appeal. [18] Acute myocardial infarction in the population over 60 years of age in Tomsk in 2008-2009 In the study of the epidemiology of myocardial infarction, the methodology of the WHO "List of Acute Myocardial Infarction" Tomsk has a higher rate of acute myocardial infarction and mortality among the population over 60 years of age. shown to be Morbidity and mortality rates are higher in men of all ages than in women, 80 except for the elderly population. By 2050, 80 percent of the elderly population will be in Europe and North
America It is estimated that by this time the number of elderly people in the Russian Federation has increased significantly, to 35 more than a million. Naturally, population growth is having a negative impact on demographic processes. [19] Epidemiological and randomized clinical trials $n-3$ polyunsaturated fatty acids ( $n-3$ PUFA) suggests that it may have a cardioprotective effect. [20] Elderly after acute myocardial infarction very long-chain $\mathrm{n}-3$ semiunsaturated fatty acid capsules in patients with standard therapy the ability to predict clinical outcomes in this population when evaluating the impact of 2-year admission considered. Based on the available literature, the calculation of sample sizes required 1,400 patients over 2 years appeared, and this $n-3$ PUFA supplement was reported to reduce cardiovascular disease by approximately $30 \%$. [21] Age from the Acute Myocardial Infarction Registry (AMIR) database - under 45 years of age and previous myocardial infarction (MI) patients with. 69 cases were studied separately in 2005-2007 and 109 cases in 20122014. Age Acute myocardial infarction in patients often develops against the background of diabetes mellitus (DM). [22; 23] Elderly in Central Asia Dependence of clinical outcomes on the course and hospitalization of acute myocardial infarction (AMI) in patients The study included 508 patients with AMI, of which 2 groups were formed: group I ( $\mathrm{n}=$ 298), the main group (MG) - Patients over 65 years of age with acute myocardial infarction of both sexes and group II $(n=210)$, control group (NG). From MG 188 patients ST 14.3\% received segmental thrombolytic therapy (TLT) with segment elevation, while in CG this figure was associated with ST segment elevation. It was $25.5 \%$ in 149 patients. Delayed medical care in elderly patients leads to 
myocardial reperfusion such as an increase in the incidence and frequency of development of heart failure as a result of a decrease in the effectiveness of TLT problems. [24] Diagnosis of ECG patients with MIST elevation in the last 15-20 years and treatment recommendations have not been republished. On the diagnosis and treatment of patients with MIST elevation the recommendations are in line with the general positions set out in the documents adopted by the International Society of Cardiologists and reflects an optimal algorithm based on modern ideas for the treatment of such patients. [25;31; 32]

\section{CONCLUSION}

Most of the risk factors for the development of cardiovascular complications in patients over 60 years of age increase with age does not increase. As an exception, a sedentary lifestyle - increases to $79.4 \%$ with age. When myocardial infarction is atypical providing highly qualified medical care as a result of nonspecialized hospitalization in $48 \%$ of patients there is no chance, resulting in high mortality, resulting in death of patients with atypical myocardial infarction is 10 times higher than in patients with typical symptoms. He has a myocardial infarction treatment of patients in appropriate conditions, pre-hospital care and continuation of treatment in a special hospital effective and correct organization of prehospital and in-hospital diagnosis, treatment and, most importantly, medical is the most optimal way to apply a single, coordinated process based on a common approach to tactical issues. Real in life, these conditions may not always be fully met. Again, the "Recommendations" for myocardial infarction we must use every opportunity to ensure that it is applied as correctly as possible, because only after that we can expect a positive result.

\section{REFERENCES}

1. E.B. Iskakov // Epidemiology of cardiovascular diseases // Medicine and ecology, 2017, 2, 19-28 pp..

2. Okrugin S.A., Kuzheleva E.A., Garganeeva A.A. WHO program "Register of acute myocardial infarction": Epidemiological monitoring of acute coronary disasters. Complex Issues of Cardiovascular Disease. 2018; 7 (1): 76-83. https://doi.org/10.17802/2306-1278-2018-71-76-83

3. I.V.Samorodskaya, S.A.Boytsov Recurrent myocardial infarction: assessment, risks, prevention. Russian journal of cardiology.2017;(6):139-145. https://doi.org/10.15829/1560-4071-2017-6139-145

4. Mustafina S.V., Winter D.A., Rymar O.D., Shcherbakova L.V., Gafarov V.V., Panov D.O., Gromova E.A., Gafarova A.V., Verevkin E G., Nikitenko T.I., Bobak M., Malyutina S.K. Obesity phenotypes and the risk of developing myocardial infarction, according to a prospective cohort study. Russian journal of cardiology. 2019; (6): 109-114. https://doi.org/10.15829/1560-4071-2019-6109-114

5. Kakorin S.V., Stogov A.V., Mkrtumyan A.M. // Acute left ventricular failure in patients with type 2 diabetes // Journal of International Journal of Interventional Cardioangiology 2015.

6. Ushkalova E.A., Zyryanov S.K., Dumchenko E.V. Approaches to antithrombotic therapy in elderly patients with atrial fibrillation. Rational Pharmacotherapy in Cardiology 201 7; 13 (2): 275-283. DOI: http://dx.doi.org/1 0.20996/18196446-2017-13-2-275-283 
7. Shu-Yun $\mathrm{Xu}$, Fong-Ling Chen, Jung-Po Liau, Jing-Yang Huang, Oswald Ndi Nfor, Dei-Yu Chao //A Matched Influenza Vaccine Strain Was Effective in Reducing the Risk of Acute Myocardial Infarction in Elderly Persons: A Population-Based Study// Medicine (Baltimore), 2016 Mar; Vol. 95 (10), pp. e2869;

8. Shesternya P.A., Shulman V.A., Nikulina S.Yu. Genetic aspects of myocardial infarction: problems and prospects. Russian journal of cardiology.2012;(1):4-9. https://doi.org/10.15829/1560-4071-2012-1133-137

9. M.A. Panteleev, F.I. Ataullakhanov // Blood coagulation: biochemical bases // Journal of Clinical oncohematology. Basic Research and Clinical Practice 2008.

10. Bolotnova T.V., Yusupov A.R., Kuimova Zh.V., Filonova M.V. // Risk factors for cardiovascular complications in patients over 60 years old. // Tyumen Medical Journal. 2014. T. 16.No. 2.P. 11-12.

11. Chumakova G.A., Pokutnev A.V., Veselovskaya N.G. Clinical features of patients after myocardial infarction with revascularization, depending on the initial status of obesity. Russian journal of cardiology. 2018; (5): 21-26. https://doi.org/10.15829/1560-4071-2018-521-26

12. A. A. Belyaev, Kotova O.V. , Akarachkova E.S. , Artemenko A.R. // Chest pain: noncardiac cardialgias in focus // RMJ "Medical Review" No. 11 of 12.25.2018 pp. 9-14

13. Garganeyeva A.A., Kuzheleva E.A., Borel K.N., Parshin E.A. // Atypical course of acute myocardial infarction: clinical and anamnestic characteristics of patients, management tactics and outcomes (according to the "Register of acute myocardial infarction"). // Cardiovascular therapy and prevention. 2016.T.15. No. 4. S.10-15.

14. Zykov M.V., Kashtalap V.V., Bykova I.S., Khryachkova O.N., Kalaeva V.V., Shafranskaya K.S., Karetnikova V.N., Barbarash O.L. Clinical and prognostic significance of serum interleukin-18 in patients with ST-segment elevation myocardial infarction. Russian journal of cardiology.2015;(11):70-74.

https://doi.org/10.15829/1560-4071-2015-1170-74

15. Pomozova T.P., Lykov Yu.V., Komarova I.S., Dyatlov N.V., Zhelnov V.V. Clinical and laboratory features of primary acute myocardial infarction in patients with obstructive and non-obstructive coronary atherosclerosis. Cardiology. 2019; 59 (10S): 41-51. https://doi.org/10.18087/cardio. 2640

16. D.E. Kuzmichev, I.M. Vil'tsev, S.V. Chirkov, R.V. Skrebov // Difficulties in diagnosing recurrent myocardial infarction in clinical practice // Problems of expertise in medicine, 2014, 44-45 pages

17. Valentina Aleksandrovna Kozik // Acute myocardial infarction with st segment elevation: markers of risk and outcomes // Abstract of dissertation for the degree of candidate of medical sciences Novosibirsk - 2019, 22 pages

18. Okrugin S.A., Garganeeva A.A., Kuzheleva E.A., Borel K.N. Assessment of the effect of a history of myocardial infarction and preinfarction state on the duration of the prehospital stage of acute myocardial infarction. Complex problems of cardiovascular diseases. 2016; (1): 55-59. https://doi.org/10.17802/2306-1278-2016-155-59 
19. Tukish O.V., Okrugin S.A., Yunusova E.Yu., Efimova E.V., Garganeyeva A.A. // Acute myocardial infarction in elderly and senile people: Epidemiological study under the WHO program "register of acute myocardial infarction" // Advances in gerontology. 2016.Vol. 29. No. 1.S. 123-127.

20. Kristian Laake, Ingebjørg Seljeflot, Erik B Schmidt, Peder Myhre, Arnljot Tveit, Harald Arnesen, Svein Solheim // Serum Fatty Acids, Traditional Risk Factors, and Comorbidity as Related to Myocardial Injury in an Elderly Population with Acute Myocardial Infarction.// J Lipids, 2016; Vol. 2016, pp. 4945720;

21. Kristian Laake, Peder Myhre, Linn M Nordby, Ingebjørg Seljeflot, Michael Abdelnoor, Pål Smith, Arnljot Tveit, Harald Arnesen, Svein Solheim// Effects of $\omega 3$ supplementation in elderly patients with acute myocardial infarction: design of a prospective randomized placebo controlled study.// BMC Geriatr, 2014 Jun 13; Vol. 14, pp. 74;

22. Nshanovna B.K., Borel K.N. Myocardial infarction in young patients: a long-term comparative analysis of developmental characteristics, clinical course and management strategy. Complex problems of cardiovascular diseases. 2016; (3). https://doi.org/10.17802/23061278-2016-3-

23. Popov S.V., Garganeeva A.A., Borel K.N., Kuzheleva E.A., Okrugin S.A. Myocardial infarction in young patients: a long-term comparative analysis of developmental characteristics, clinical course and management strategy. Complex problems of cardiovascular diseases. 2016;(4):66-72.

https://doi.org/10.17802/2306-1278-2016-4$66-72$
24. Nikishin A.G., Kurbanov R.D., Pirnazarov M.M. Time of hospitalization and outcomes of acute myocardial infarction in elderly patients in the Central Asian region. Cardiovascular therapy and prevention. 2012; 11 (2): 53-56. https://doi.org/10.15829/1728-8800-2012-253-56

25. Chairman - Prof. Ruda M.Ya. prof. Golitsyn S.P., prof. Gratsianskiy N.A., Ph.D. Komarov A.L., prof. Panchenko E.P., MD Staroverov I.I., prof. Tereshchenko S.N., MD Yavelov IS // Diagnostics and treatment of patients with acute myocardial infarction with ST segment elevation ECG * Diagnostics and treatment of patients with acute myocardial infarction with ST segment elevation ECG * // * Recommendations published in the journal "Cardiovascular Therapy and Prevention" 2007; 6 (8), 415500 pages

26. Alikhanova K.A., Omarkulov B.K., Abugalieva T.O., Zhakipbekova V.A. // Study of the prevalence of diseases of the cardiovascular system among the population of the Karaganda region. 2013. No. 9-5. S. 804-809.

27. Kurbanov R.D., Nikishin A.G., Pirnazarov M.M., Khasanov M.S., Nurbaev T.A., Yakubbekov N.T., Abdullaeva S.Ya. // Prognostic value of laboratory and instrumental diagnostics results in patients with acute myocardial infarction on the background of diabetes mellitus. // Eurasian Journal of Cardiology. 2013. No. 2. S. 27-35.

28. Garganeeva A.A., Tukish O.V., Kuzheleva E.A. // Long-term survival of elderly and senile patients after myocardial infarction, depending on the management tactics in the acute period 
of the disease. // Advances in gerontology. 2017. No. 5.P.709-715.

29. Ryzhova T.A. // Features of the course of myocardial infarction in elderly and senile women. // Abstract of the thesis. dis. for a job. uch. step. Cand. honey. sciences. Kemerovo, 2013-26 p.

30. Nikishin A.G., Pirnazarov M.M., Yakubbekov N.T., Abdullaeva S.Ya., Khasanov M.S., etc. // Predictor role of spectral indicators of heart rate variability in patients with acute myocardial infarction on the background of diabetes mellitus. // Medical News. 2016. No. 1 (256). P. 63-66.

31. Rakhmatova D.B. «Main» Symptoms and leading clinical options for the flow of acute coronary syndromes in women. //Asian Journal of Multidimensional Research (AJMR) - 2019, Volume: 8, Issue :11. page 69-74. DOI: 10.5958 / 22784853.2019.00307.0

32. Rakhmatova D.B. "Main" symptoms and leading clinical variants of the course of acute coronary syndrome in women. // Journal of Biomedicine and Practice 2019, vol. 2, issue 6, page 68-73. http: // dx.doi.org/10.26739/2181-9300-2019-6-8

33. Rakhmatova D.B. Analysis of the risk factors of Chd in persons over 60 years among the population of the city of Bukhara. //Asian studies, India - 2019, no.1. page 33-38, https://journals.eduindex.org/index.php/a s/article/view/4252/2469

34. Rakhmatova D.B., Razhabova G.Kh., Mavlonov N.Kh. Analysis of risk factors for coronary heart disease in people over 60 years old among the population of the city of Bukhara. // New day in Medicine 2018. No.4(24). page 102-104, https://bsmi.uz/images/material_2019/yan var/jurnal/2018-4.pdf 